\title{
РАСЧЕТ СОСТАВА ТРОЙНОГО АЗЕОТРОПА ПРИ ПОСТОЯННОМ ДАВЛЕНИИ
}

При разделении смесей, основанном на использовании равновесия между жидкостью и паром, большую роль играют азеотропные характеристики систем. Весьма важное значение имеет при этом надежная оценка указанных характеристик по легко доступным исходным данным. Известные методы количественной оценки дают часто весьма приближенные результаты и, как правило, применимы лишь к гомоазеотропным системам.

Ранее [1] для расчета состава тройного азеотропа любого типа предложен метод, где точка, отвечающая составу тройного азеотропа, рассматривалась как точка пересечения трех кривых $\alpha_{i k}=1$ *, связывающих ее с точками составов бинарных азеотропов $\left(x_{i}^{i k}\right)$. Расположение проекций указанных кривых на треугольнике составов описывается уравнениями типа

$$
x_{i(i k)}=\frac{x_{i}}{1-x_{j}}=x_{i}^{i k}+C_{i k} x_{j},
$$

совместное решение которых позволяет определить состав тройного азеотропа. Решение может быть осуществлено как графическим, так и аналитическим путем, например методом Ньютюна.

* Принятые обозначения:

$\alpha_{i h}=\frac{y_{i} / y_{k}}{x_{i} / x_{h}} \cdots$ относительная летучесть компонентов $i$ и $k$,

$y$ - молярная доля в паре,

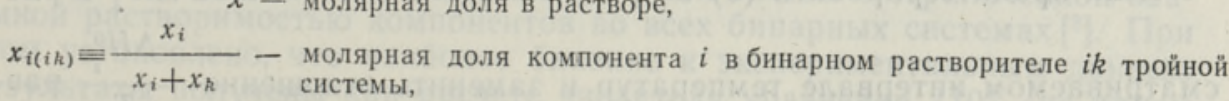
$P$ - давление,

$P_{i}$ - давление пара компонента $i$,

$T$ - температура, $\mathrm{K}$,

$t$ - температура, ${ }^{\circ} \mathrm{C}$,

$\Delta H_{i}^{0}$ - теплота испарения компонента $i$,

$\gamma_{i}-$ коэффициент активности компонента $i$. 
В настоящем сообщении приводится программа, записанная на алгоритмическом языке ФОРТРАН, для расчета состава тройного азеотропа на ЭВМ. Исходная информация, необходимая для этого расчета, включает данные о составах бинарных азеотропов и значения констант $C_{i k}$.

Значение константы может быть приближенно вычислено по полным данным о равновесии жидкость-пар в бинарных системах $\left[{ }^{2}\right]$

$$
C_{i k} \approx-\frac{\lg \alpha_{i j}^{(j)}-\lg \alpha_{k j}^{(j)}}{\lg \alpha_{i k}^{(i)}-\lg \alpha_{i k}^{(k)}}
$$

Если же предположить, что пар подчиняется законам идеальных газов и что предельное значение коэффициента активности может быть определено из соотношения

$$
\lg \gamma_{i}^{(j)} \approx \frac{\lg \gamma_{i}^{i j}}{x_{j}^{i j}}
$$

то значение константы может быть рассчитано по данным о бинарных азеотропах и компонентах

$$
C_{i k} \approx-\frac{\lg \left(\frac{P_{k}^{0}}{P_{i}^{0}}\right)^{(j)}-\left(\frac{\lg P / P_{i}^{0}}{x_{j}^{i j}}\right)^{i j}+\left(\frac{\lg P / P_{k}^{0}}{x_{j}^{k j}}\right)^{a 3}}{\lg \left(\frac{P_{i}^{0}}{P_{k}^{0}}\right)^{(k)}+\left(\frac{\lg P / P_{k}^{0}}{x_{i}^{i k}}+\frac{\lg P / P_{i}^{0}}{x_{k}^{i k}}\right)^{i k}-\lg \left(\frac{P_{i}^{0}}{P_{k}^{0}}\right)^{(i)}} .
$$

Формулу (4) можно использовать для расчета при постоянной температуре и постоянном давлении.

Так как на практике чаще всего приходится иметь дело с разделением смесей в изобарических условиях, в настоящем сообщении дадим вывод формулы для расчета константы $C_{i k}$, в ходе которого логарифмы давлений паров компонентов и бинарных азеотропов заменим соответственно температурами кипения, величинами более доступными при постоянном давлении.

Температурная зависимость давлений паров компонентов может быть выражена уравнением

$$
\ln P / P_{i}^{0}=\int_{T}^{T i} \frac{\Delta H_{i}^{0}}{R T^{2}} d T
$$

где $T_{i}$ - температура кипения компонента $i$ при давлении $P$, полученным из уравнения Клаузиуса-Клапейрона.

Если проинтегрировать (5) в предположении постоянства $\Delta H_{i}^{0}$ в рассматриваемом интервале температур и заменить отношение $\frac{\Delta H^{0}}{T}$ равной ему энтропией испарения компонента $\Delta S_{i}^{0}$, получим следующие выражения для логарифмов отношений давлений паров

$$
\lg \left(P / P_{i}^{0}\right)^{i k}=\frac{\Delta S_{i}^{0}\left(T_{i}-T_{i k}\right)}{2,303 R T_{i k}},
$$




$$
\lg \left(P_{i}^{0} / P_{k}^{0}\right)^{(j)}=\frac{\Delta S_{k}^{0} T_{k}-\Delta S_{i}^{0} T_{i}+T_{j}\left(\Delta S_{i}^{0}-\Delta S_{k}^{0}\right)}{2,303 R T_{j}},
$$

которые подставим в формулу (4), дав индексам цифровые значения $i=1, k=2$ и $j=3$.

$$
\begin{gathered}
\frac{\Delta S_{1}^{0} T_{1}-\Delta S_{2}^{0} T_{2}^{2}+T_{3}\left(\Delta S_{2}^{0}-\Delta S_{1}^{0}\right)}{T_{3}}+ \\
+\frac{\Delta S_{1}\left(T_{13}-T_{1}\right)}{T_{13} \cdot x_{3}^{13}}-\frac{\Delta S_{2}^{0}\left(T_{23}-T_{2}\right)}{T_{23} \cdot x_{3}^{23}} \\
-\frac{\Delta S_{1}^{0}\left(T_{2}-T_{1}\right)}{T_{2}}+\frac{\Delta S_{1}^{0}\left(T_{1}-T_{12}\right)}{T_{12} \cdot x_{2}^{12}}- \\
-\frac{\Delta S_{2}^{0}\left(T_{12}-T_{2}\right)}{T_{12} \cdot x_{1}^{12}}-\frac{\Delta S_{2}^{0}\left(T_{2}-T_{1}\right)}{T_{1}}
\end{gathered}
$$

Предположение о равенстве энтропий испарения компонентов $\left(\Delta S_{1}^{0}=\Delta S_{2}^{0}\right)$ позволяет выразить $C_{12}$ через легко доступные исходные данные

$$
C_{12} \approx-\frac{\frac{T_{1}-T_{2}}{T_{3}}+\frac{T_{13}-T_{1}}{T_{13} x_{3}^{13}}-\frac{T_{23}-T_{2}}{T_{23} x_{3}^{23}}}{\frac{T_{2}-T_{1}}{T_{2}}+\frac{T_{1}-T_{12}}{T_{12} x_{2}^{12}}-\frac{T_{12}-T_{2}}{T_{12} x_{1}^{12}}-\frac{T_{2}-T_{1}}{T_{1}}} .
$$

Полученную формулу можно, однако, еще более упростить, если предположить, что равенство температур в знаменателях отдельных членов более чем на порядок больше разности этих температур в числителях. После сокращения константу $C_{12}$ можно выразить через температуры кипения компонентов и бинарных азеотропов, ${ }^{\circ} \mathrm{C}$

$$
C_{12} \approx-\frac{t_{1}+\frac{t_{13}-t_{1}}{x_{3}^{13}}-t_{2}-\frac{t_{23}-t_{2}}{x_{3}^{23}}}{\frac{t_{1}-t_{12}}{x_{2}^{12}}-\frac{t_{12}-t_{2}}{x_{1}^{12}}} .
$$

Формула (10) выведена иным путем для систем с ограниченной взаимной растворимостью компонентов во всех бинарных системах $\left[{ }^{3}\right]$. При этом установлено, что наиболее близкие к экспериментальным данным результаты получены при расчете числителя уравнения (10) «на одном уровне», т. е. при практически одинаковом содержании третьего компонента $\left(x_{3}^{13} \approx x_{3}^{23}\right)$.

В настоящем сообщении расчет проведен на примере тройного гетероазеотропа тиофен (1)-вода (2)-изопропанол (3), состав и температура кипения которого определены для давлений $760,600,400$ и 200 мм рт.ст. Методика исследования описана в [ $\left.{ }^{4}\right]$. Результаты экспериментального исследования бинарных азеотропов приведены в табл. 1. 
Таблица 1

Зависимость температур кипения и составов бинарных азеотропов от давления

\begin{tabular}{|c|c|c|c|c|c|c|c|c|}
\hline \multirow{2}{*}{$\begin{array}{c}\text { Бинарный } \\
\text { азеотроп }\end{array}$} & \multicolumn{2}{|c|}{760 мм рт.ст. } & \multicolumn{2}{|c|}{600 ми рт. ст. } & \multicolumn{2}{|c|}{400 мм рт. ст. } & \multicolumn{2}{|c|}{200 м. рт. ст. } \\
\hline & $\begin{array}{l}\text { т. кип., } \\
{ }^{\circ} \mathrm{C}\end{array}$ & $x_{1}^{12}$ & $\begin{array}{l}\text { т. кип., } \\
{ }^{\circ} \mathrm{C}\end{array}$ & $x_{1}^{12}$ & $\begin{array}{l}\text { т. кип., } \\
{ }^{\circ} \mathrm{C}\end{array}$ & $\begin{array}{c}x_{1}^{12} \\
1\end{array}$ & $\begin{array}{l}\text { т. кип., } \\
{ }^{\circ} \mathrm{C}\end{array}$ & $x_{1}^{12}$ \\
\hline $\begin{array}{l}\text { Тиофен (1) - } \\
\text {-вода (2) }\end{array}$ & 72,3 & 0,674 & 65,9 & 0,682 & 55,7 & 0,703 & 39,0 & 0,740 \\
\hline $\begin{array}{l}\text { Тиофен (1)- } \\
\text {-изопро- } \\
\text { панол (2) }\end{array}$ & 74,2 & 0,534 & 67,4 & 0,556 & 57,2 & 0,591 & 41,1 & 0,644 \\
\hline $\begin{array}{c}\text { Изопропа- } \\
\text { нол (1)- } \\
\text {-вода (2) }\end{array}$ & 80,1 & 0,687 & 74,2 & 0,682 & 65,1 & 0,676 & 50,2 & 0,671 \\
\hline
\end{tabular}

Эти данные, а также значения температур кипения компонентов, рассчитанные для указанных давлений по уравнению Антуана, константы которого приведены в табл. 2 , использованы для расчета констант $C_{i k}$ по формуле (10) при определении состава тройного азеотропа.

таблица 2

Константы уравнения Антуана

\begin{tabular}{l|c|c|c|c}
\hline \multicolumn{1}{c|}{ Компонент } & $A$ & $B$ & $C$ & Литература \\
\hline Тиофен & 6,9593 & 1246,04 & 221,35 & {$\left[^{5}\right]$} \\
Вода & 7,9668 & 1668,2 & 228 & {$\left[{ }^{6}\right]$} \\
Изопропанол & 7,7668 & 1371,6 & 198,5 & {$\left[{ }^{7}\right]$}
\end{tabular}

Справедливость сделанных при выводе формулы (10) предположений проверена путем графического сопоставления кривых $\alpha_{i k}=1$. Эти кривые, полученные по уравнению (1), константы которого рассчитаны по формулам (4) и (8)-(10), изображены на рисунке сплошными линиями с указанием номера формулы. Прерывистой линией обозначены «исправленные» кривые, числитель константы которых вычислялся по формуле (10) «на уровне» состава азеотропа $x_{3}^{13}$, т. е. с привлечением данных о равновесии жидкость-пар в системах $2-3\left[{ }^{8}\right]$ и $1-2$. Значения констант приведены в табл. 3.

Таблица 3

\begin{tabular}{c|r|r|r|r|c}
\hline \multirow{2}{*}{$C_{i k}$} & \multicolumn{5}{|c}{ Результаты расчета $C_{\text {ik }}$ по формулам } \\
\cline { 2 - 6 } & $(4)$ & $(8)$ & $(9)$ & $(10)$ & $(10)$ \\
\hline & 0,072 & 0,070 & 0,108 & 0,106 & $-0,074^{*}$ \\
$C_{12}$ & $-0,519$ & $-0,746$ & $-0,773$ & $-0,758$ & $-0,758$ \\
$C_{31}$ & 0,225 & 0,167 & 0,157 & 0,138 & $0,432^{*}$ \\
$C_{23}$ & & & &
\end{tabular}

* Числитель в уравнении (10) рассчитан для $x_{3}^{13} \approx x_{3}^{23}$.

Из данных рисунка следует, что наибольшее расхождение наблюдается в ходе кривых $\alpha_{i k}=1$ с наиболее отдаленной исходной точкой. 


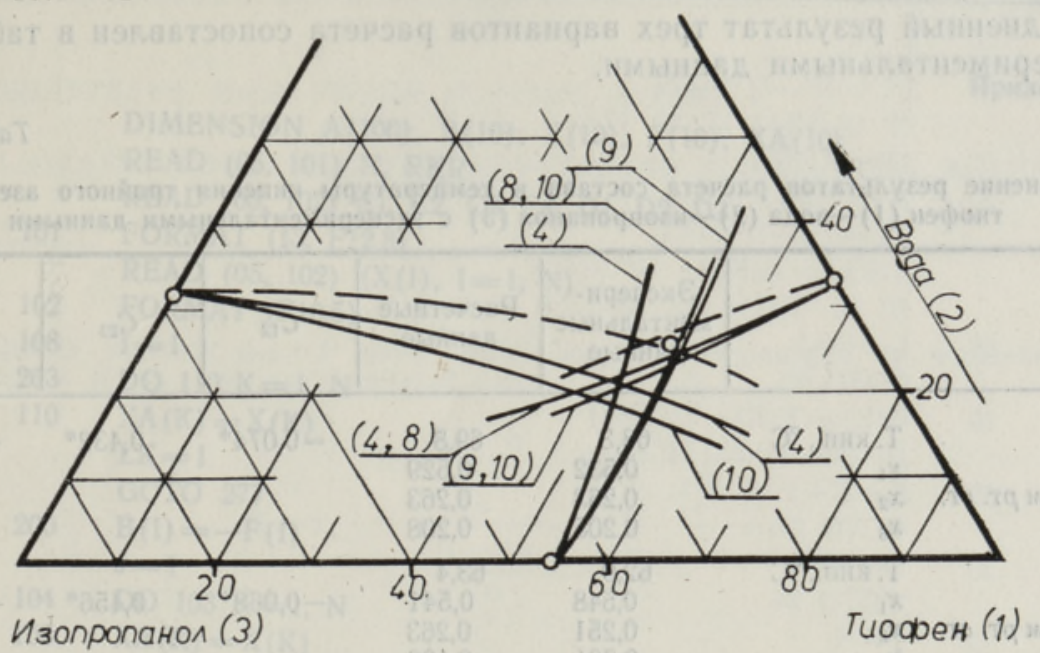

Расположение кривых $\alpha_{i k}=1$, константы $C_{i k}$ уравнений которых рассчитаны по формулам (4), (8), (9) и (10).

О - экспериментальные данные.

Расчет $C_{12}$ и $C_{23}$ по формуле (4) дал несколько более точные результаты, чем расчет по остальным формулам**. Менее точный результат расчета $C_{31}$ по указанной формуле объясняется, видимо, непригодностью уравнения Антуана для экстраполяции значений $P_{i}^{0}$ на температуру, значительно превышающую температуру кипения компонента.

Наиболее близкими к точке тройного азеотропа являются точка пересечения кривых $\alpha_{i k}=1$, выходящих из ближайших бинарных азеотропов, и точка пересечения «исправленных» кривых. Уравнения, полученные для последних, использовали при определении состава тройного азеотропа.

Состав рассчитан на ЭВМ «Видеотон 1010 Б». По программе (см. приложение) совместно решались два уравнения

$$
\begin{aligned}
& C 1 Y^{2}+C 2 Y Z+C 1 Z^{2}+C 3 Y+C 4 Z=0 \quad \text { и } \\
& D 1 Y^{2}+D 2 Y+Z+D 3=0,
\end{aligned}
$$

где $C 1=C_{i k}, \quad C 2=2 C_{i k}, \quad C 3=1-x_{i}^{i k}-C_{i k}, \quad C 4=x_{i}^{i k}+C_{i k}, \quad D 1=C_{k j}$, $D 2=x_{k}^{k j}-C_{k j}, \quad D 3=x_{k}^{k j}, \quad Y=x_{i}, \quad Z=x_{k}$

Для первого варианта расчета принято $i=1, k=2$ и $j=3$. Два остальных варианта получены путем замены индексов согласно схеме $\swarrow_{1} \triangle_{2}^{3}$.

** Значения энтропий испарения $\left(\Delta S_{1}^{0}=20,97, \Delta S_{2}^{0}=26,04\right.$ и $\Delta S_{3}^{0}=31,09$

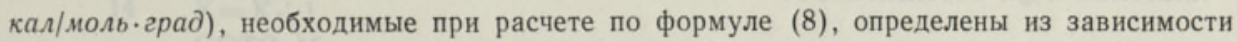
$\Delta S_{i}^{0}=\Delta H_{i}^{0} / T_{i}$, значения $\Delta H_{i}^{0}$ в которой для воды и спирта заимствованы из [5], а для тиофена вычислены по формуле $\Delta H_{i}^{0}=2,303 B R T^{2} \Delta Z /\left(C+t_{i}\right)^{2}$. Для расчета $\Delta Z$ использовали формулу Хаггенмахера [ $\left.{ }^{9}\right]$. 
Усредненный результат трех вариантов расчета сопоставлен в табл. 4 с экспериментальными данными.

Таблица 4

Сравнение результатов расчета состава и температуры кипения тройного азеотропа тиофен (1)-вода (2)-изопропанол (3) с экспериментальными данными

\begin{tabular}{|c|c|c|c|c|c|c|}
\hline & & $\begin{array}{c}\text { Экспери- } \\
\text { ментальные } \\
\text { данные }\end{array}$ & $\begin{array}{c}\text { Расчетные } \\
\text { данные }\end{array}$ & $C_{12}$ & $C_{23}$ & $C_{31}$ \\
\hline 760 мм рт. ст. & $\begin{array}{l}\text { T. кип., }{ }^{\circ} \mathrm{C} \\
x_{1} \\
x_{2} \\
x_{3}\end{array}$ & $\begin{array}{c}68,3 \\
0,532 \\
0,263 \\
0,205\end{array}$ & $\begin{array}{c}69,8 \\
0,529 \\
0,263 \\
0,208\end{array}$ & $-0,074^{*}$ & 0,432 * & $-0,758$ \\
\hline 600 мм рт. ст. & $\begin{array}{l}\text { T. кип., }{ }^{\circ} \mathrm{C} \\
x_{1} \\
x_{2} \\
x_{3}\end{array}$ & $\begin{array}{c}62,9 \\
0,548 \\
0,251 \\
0,201\end{array}$ & $\begin{array}{c}63,4 \\
0,541 \\
0,263 \\
0,196\end{array}$ & $-0,068^{*}$ & $0,456^{*}$ & $-0,712$ \\
\hline 400 мм рт. ст. & $\begin{array}{l}\text { T. кип., }{ }^{\circ} \mathrm{C} \\
x_{1} \\
x_{2} \\
x_{3}\end{array}$ & $\begin{array}{l}52,8 \\
0,574 \\
0,237 \\
0,189\end{array}$ & $\begin{array}{c}53,4 \\
0,571 \\
0,249 \\
0,180\end{array}$ & $-0,094^{*}$ & $0,412^{*}$ & $-0,754$ \\
\hline 200 мм pт. $c T$. & $\begin{array}{l}\text { T. кип., }{ }^{\circ} \mathrm{C} \\
x_{1} \\
x_{2} \\
x_{3}\end{array}$ & $\begin{array}{l}36,7 \\
0,614 \\
0,218 \\
0,168\end{array}$ & $\begin{array}{c}37,5 \\
0,609 \\
0,220 \\
0,171\end{array}$ & $-0,081^{*}$ & $0,357^{\text {* }}$ & $-0,691^{*}$ \\
\hline
\end{tabular}

Температура кипения тройного азеотропа рассчитана также с использованием значений констант, приведенных в табл. 4, по формуле

$$
t_{123}=t_{12}+\left(\frac{t_{1}-t_{12}}{x_{2}^{12}} \cdot C_{12}+t_{1}+\frac{t_{13}-t_{1}}{x_{3}^{13}}-t_{12}\right) x_{3},
$$

предложенной в $\left[{ }^{10}\right]$.

ЛИ ТЕРА Т У РА

1. Kudrjawzewa, L. S., T o ome, M. J., Sussarjew, M. P., Mh. Chem., 105, 334 (1974).

2. Kudr jawzewa, L. S., Kirs s, H. H., E ise n, O. G., Mh. Chem., 105, 19 (1974).

3. С у с а ре в М. П., К удря вце в а Л. С., Ж. физ. химии, 48, 1945 (1974).

4. К удря вц е в Л. С., Т оом е М. Ю., Эй зен О. Г., Ж. прикл. химии, 48, 1047 (1975).

5. Справочник химика, 1. Л.-M., 1962.

6. Holmes, M. J., van W in kle, M., Ind. Engng Chem., 62, 21A (1970).

7. B r ow n, I., Fock, W., S m i th, F., Austral. J. Chem., 9, 364 (1956).

8. Коган В. Б., Фр ид м а В В. М., Кафар ов В. В., Равновесие между жидкостыю и паром. М., 1966.

9. H a g g e n m a cher, J. E., J. Amer. Chem. Soc., 68, 1633 (1946).

10. Тооме М. Ю., Кудря вцева Л. С., Эй зен О. Г., Ж. прикл. химин, 49, 863 (1976).

Ннститут химии

Академии наук Эстонской ССР
Поступила в редакцию 23/II 1976 


\section{Приложение}

DIMENSION A(100), B(10), X(10), F(10), XA(10)

READ $(05,101) \mathrm{N}, \mathrm{RKR}$

READ $(05,102) \mathrm{C} 1, \mathrm{C} 2, \mathrm{C} 3, \mathrm{C} 4, \mathrm{D} 1, \mathrm{D} 2, \mathrm{D} 3$

101

FORMAT (I3, F12.8)

$\operatorname{READ}(05,102)(X(I), I=1, N)$

102 FORMAT (F10.5)

$108 \quad \mathrm{I}=1$

203 DO $110 \mathrm{~K}=1, \mathrm{~N}$

$110 \quad \mathrm{XA}(\mathrm{K})=\mathrm{X}(\mathrm{K})$

$\mathrm{LK}=1$

GOTO 277

200

$\mathrm{B}(\mathrm{I})=-\mathrm{F}(\mathrm{I})$

$\mathrm{J}=1$

104 DO $103 \mathrm{~K}=1, \mathrm{~N}$

$103 \quad \mathrm{XA}(\mathrm{K})=\mathrm{X}(\mathrm{K})$

$\mathrm{XA}(\mathrm{J})=\mathrm{XA}(\mathrm{J})+0.01 * \mathrm{XA}(\mathrm{J})$

$\mathrm{LK}=2$

GOTO 277

$201 \quad \mathrm{~A}((\mathrm{I}-1) * \mathrm{~N}+\mathrm{J})=(\mathrm{F}(\mathrm{I})+\mathrm{B}(\mathrm{I})) /(0,01 * \mathrm{X}(\mathrm{J}))$

$\mathrm{J}=\mathrm{J}+1$

IF $(\mathrm{J}-\mathrm{N})$ 104, 104, 202

$202 \quad \mathrm{I}=\mathrm{I}+1$

IF $(\mathrm{I}-\mathrm{N})$ 203, 203, 204

$204 \quad \mathrm{I}=1$

$24 \quad \mathrm{II}=(\mathrm{I}-1) * \mathrm{~N}+\mathrm{I}$

IF (A(II)) 3, 4, 3

$4 \quad \mathrm{~K}=\mathrm{I}+1$

$\mathrm{KI}=(\mathrm{K}-1) * \mathrm{~N}+\mathrm{I}$

IF (A(KI)) 5, 4, 5

$5 \quad \mathrm{~L}=\mathrm{I}$,

$8 \mathrm{IL}=(\mathrm{I}-1) * \mathrm{~N}+\mathrm{L}$

$\mathrm{KL}=(\mathrm{K}-1) * \mathrm{~N}+\mathrm{L}$

$\mathrm{TP}=\mathrm{A}(\mathrm{IL})$

$\mathrm{A}(\mathrm{IL})=\mathrm{A}(\mathrm{KL})$

$\mathrm{A}(\mathrm{KL})=\mathrm{TP}$

$6 \quad \mathrm{~L}=\mathrm{L}+1$

GOTO 8

$7 \quad \mathrm{TP}=\mathrm{B}(\mathrm{I})$

$\mathrm{B}(\mathrm{I})=\mathrm{B}(\mathrm{K})$

$\mathrm{B}(\mathrm{K})=\mathrm{TP}$

3 IF (A(II)-1.0) 9, 10, 9

10 IF $(\mathrm{I}-\mathrm{N}) \quad 11,12,11$

$9 \quad \mathrm{TP}=\mathrm{A}(\mathrm{II})$

$\mathrm{K}=\mathrm{I}$

$15 \mathrm{IK}=(\mathrm{I}-1) * \mathrm{~N}+\mathrm{K}$

$\mathrm{A}(\mathrm{IK})=\mathrm{A}(\mathrm{IK}) / \mathrm{TP}$

IF $(\mathrm{K}-\mathrm{N}) 14,13,14$

$14 \quad \mathrm{~K}=\mathrm{K}+1$

GOTO 15

$13 \quad \mathrm{~B}(\mathrm{I})=\mathrm{B}(\mathrm{I}) / \mathrm{TP}$

GOTO 10

$11 \mathrm{~K}=\mathrm{I}+1$ 


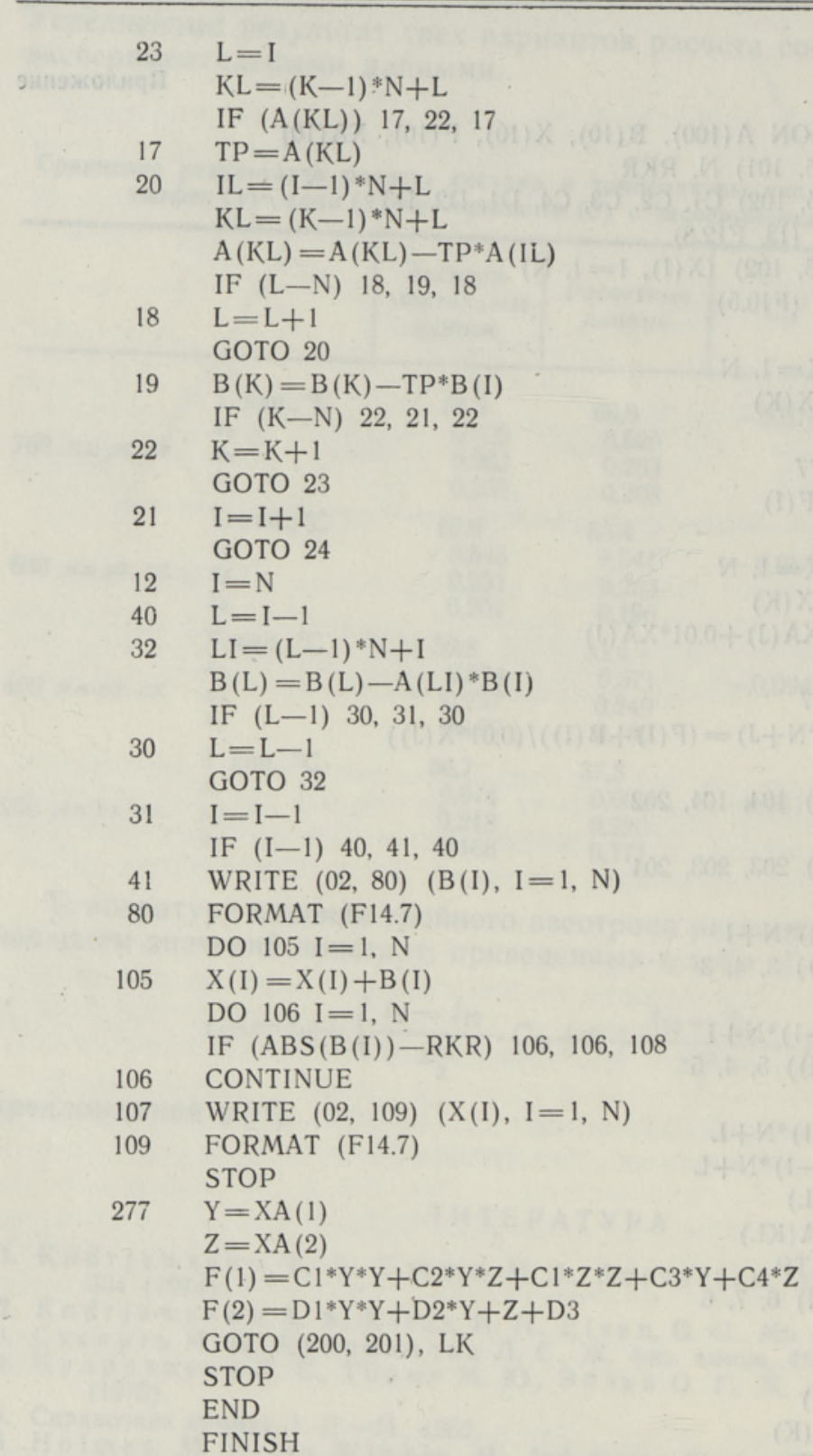

Обозначения исходной информации программы:

$\mathrm{N}$ - число уравнений, RKR - точность решения, C1, C2, C3, C4, D1, D2, D3 коэффициенты системы уравнений $(11), \mathrm{X}(1-\mathrm{N})$ - начальное решение. 
L. KUDRJAVTSEVA, Merike TOOME, O. EISEN, R. TAHT

\section{KOLMIKASEOTROOBI KOOSTISE ARVUTAMINE PUSIVA RÖHU KORRAL}

Võrrandis

$$
x_{i(i k)}=x_{i}^{i k}+C_{i k} x_{j},
$$

mis kirjeldab kõverate $\alpha_{i h}=1$ käiku Gibbsi koostiste kolmnurgal, on konstandi $C_{i k}$ arvutamiseks püsiva rōhu korral esitatud valem

$$
C_{i k} \approx-\frac{t_{1}+\frac{t_{13}-t_{1}}{x_{3}^{13}}-t_{2}-\frac{t_{23}-t_{2}}{x_{3}^{23}}}{\frac{t_{1}-t_{12}}{x_{2}^{12}}-\frac{t_{12}-t_{2}}{x_{1}^{12}}} .
$$

Esitatakse kolmikaseotroobi koostise raalimise programm. Programmeerimiskeeleks on valitud FORTRAN, lähteinformatsioon on saadud kaksikaseotroopide andmeist ja konstandi $C_{i k}$ väärtustest.

Arvutust illustreeritakse kolmikaseotroobi tiofeen(1)-vesi(2)-isopropanool(3) näite varał, mille koostis ja keemistemperatuur olid määratud rōhul $760,600,400$ ja $200 \mathrm{~mm} \mathrm{Hg}$.

L. KUDRJAWZEWA, Merike TOOME, O. EISEN, R. TÄHT

\section{ZUR BERECHNUNG DER ZUSAMMENSETZUNG TERNÄRER AZEOTROPE BEI KONSTANTEM DRUCK}

Es wird eine Formel hergeleitet, mit deren Hilfe man die Konstante $C_{i k}$ der Gleichung der $\alpha_{i k}=1$-Kurve

$$
x_{i(i k)}=x_{i}^{i k}+C_{i k} x_{j}
$$

an Hand von leicht zugänglichen Daten berechnen kann.

Für die Lösung des Systems dieser Gleichungen, die zur Ermittlung der Zusammensetzung des ternären Azeotrops führt, wird ein Programm in Programmiersprache FORTRAN erarbeitet. Als Ausgangsdaten werden dabei Werte der Konstanten $C_{i k}$ und der Zusammensetzung der binären Azeotrope benutzt.

Am Beispiel des Azeotrops Thiophen-Wasser-Propanol wird im Vergleich zu den bei mehreren Drucken experimentell erhaltenen Werten die Berechnungsmethode illustriert. 